\title{
Giant Hepatic Hydatid Cyst with Retroperitoneal Development-Management of Liver Hydatidosis
}

\author{
Marius Murariu ${ }^{1,2, *}$, Sabin Pop ${ }^{1,2}$ and Sorin Olariu ${ }^{1,2}$ \\ ${ }^{\text {I }}$ Department of Surgery, First Clinic of Surgery, "Victor Babeș” University of Medicine and Pharmacy Timișoara, \\ Eftimie Murgu Sq. no. 2, 30004I Timișoara, Romania; sabinpop@yahoo.com (S.P.); olariu.sorin@umft.ro (S.O.) \\ ${ }^{2}$ First Clinic of Surgery, "Pius Brînzeu” Emergency County Hospital Timişoara, Liviu Rebreanu Bv. I56, \\ 300723 Timişoara, Romania \\ *Correspondence: murariu.marius@umft.ro; Tel.: +40-748331286
}

Submitted: 8 February 202I; Accepted: I8 February 202I; Published: 22 February 202I

\begin{abstract}
I) Background: Infection with Echinococcus granulosus is the cause of hydatid cysts located frequently in the liver and lungs, but also in the kidneys, bones, spleen, brain and muscles. Cystic echinococcosis is sometimes complicated to treat, requiring radical surgery and prolonged medication. The hydatid cyst in the retroperitoneal space is frequently asymptomatic, unless it grows and produces symptoms caused by high intracystic pressure, secondary infection, wall breaking and allergy. (2) Case Report: We present the case of a 47-year old male patient, admitted for a giant bilobed retroperitoneal hydatid cyst. Following diagnosis, the patient was operated on using an anterior midline abdominal approach. The cyst was inactivated, the contents were evacuated and the cavity was drained and cleaned by opening the cyst in the middle area and at its lower pole, respectively. Subsequently, the cyst required re-installation of the drain. The evolution of the case was finally favorable but confirmed that the postoperative evolution of large hydatid cysts can be unexpected and even followed by complications. (3) Conclusions: For the presented case, the classic surgical method was the appropriate choice, considering the large size of the hydatid cyst. Bilobed cysts and those with calcified walls may cause problems with the spontaneous closing of the remaining cavity. For complicated cysts, the treatment is radical or conservative surgery, while for uncomplicated cysts, minimally invasive treatment is an appropriate choice.
\end{abstract}

Keywords: echinococcosis; hydatid cyst; hepatic; retroperitoneum

How to cite: Murariu, M.; Pop S.; Olariu, S. Giant Hepatic Hydatid Cyst with Retroperitoneal Development-Management of Liver Hydatidosis. Timisoara Med. 2020, 2020(2), 9; doi:I0.35995/tmj20200209.

\section{Background}

Echinococcosis is a parasitic disease caused by the larvae of E. granulosus [I]. It occurs more frequently in "endemic" areas. It is significant in frequency in Mediterranean countries, Eastern Europe, South America and in the Far East [2]. E. granulosus is endemic in countries where farming is the usual occupation of the population $[3,4]$. The infection occurs frequently in people who come into contact with dogs, but also in 
those who have certain professions more frequently affected by this parasitosis: animal breeders, shepherds and butchers.

In the intestine of dogs, in general, lives the adult Taenia echinococcus granulosus, and eggs are excreted in the feces and spread on the ground, grass, water, fruits and vegetables. Then, the eggs are ingested incidentally by the intermediate host (sheep, pig or human).

Humans may infect themselves accidentally, either by eating infested and unwashed vegetables / fruits, or from the dog's contaminated fur. In the human intestine, under the action of duodenal juice, the shell dissolves and releases the hexacant embryos that pass through the intestinal wall into the portal circulation. Hydatid cysts are located frequently in the liver $(60-70 \%)$ and lungs $(20-30 \%)$, and not so frequently in the kidneys, bones, spleen, brain and muscles [2]. Hydatid cysts are seen more frequently at young ages, up to 40 years. The infection occurs during the childhood period and grows very slowly (a possible $\mathrm{I}-3 \mathrm{~cm}$ per year), taking many years to have a significant dimension [5]. The disease evolves asymptomatically for many years until the clinical signs appear because of the growth of the hydatid cysts. The clinical symptoms depend on the position of the cyst, and they are not specific.

Hydatid cysts of retroperitoneum are especially rare, and there are few cases reported in the literature. Cases with large or giant retroperitoneal cysts are even rarer, and as a result, the surgical removal of such cysts can be difficult. According to the World Health Organization, hydatid cysts over Io $\mathrm{cm}$ are considered large $[6,7]$. Cysts over $15 \mathrm{~cm}$ are considered giant.

Hydatid cysts in the retroperitoneal space are frequently asymptomatic until they grow and produce symptoms. Ultrasonography, computed tomography and magnetic resonance are the techniques usually used for the diagnosis of hydatid cyst. Treatment for cystic echinococcosis is sometimes difficult because it could require radical surgery and prolonged medication.

The aim of our paper is to present a rare case, where the hydatid cyst was formed in the left hepatic lobe and evolved for a long time completely retroperitoneal towards the lower part of the abdomen.

\section{Case Report}

\section{Presentation}

We present the case of a 47-year old male patient, who was admitted to our surgery department for a giant retroperitoneal tumor. His symptoms included bloating and a persistent sensation of pressure in the abdomen. The patient was diagnosed with type II diabetes mellitus.

\section{Diagnosis}

Ultrasonography revealed an intraabdominal tumoral mass, which initially was mistakenly diagnosed as a pancreatic tumor. Abdominal computed tomography with contrast medium revealed a bilobed cystic mass with partially calcified walls, in close contact with the spleen, the pancreatic tail and the great gastric curvature, measuring $23 / 13 / 14 \mathrm{~cm}$, and containing internal daughter cysts (Figure I). The cyst was connected to the liver at the left border of the left hepatic lobe. The mass belonged to the left hepatic lobe, representing a hepatic hydatid cyst with daughter cysts. There were no other cystic structures in the abdomen. The pancreas had a normal structure, and the kidneys, spleen and adrenal glands were also of normal appearance. 

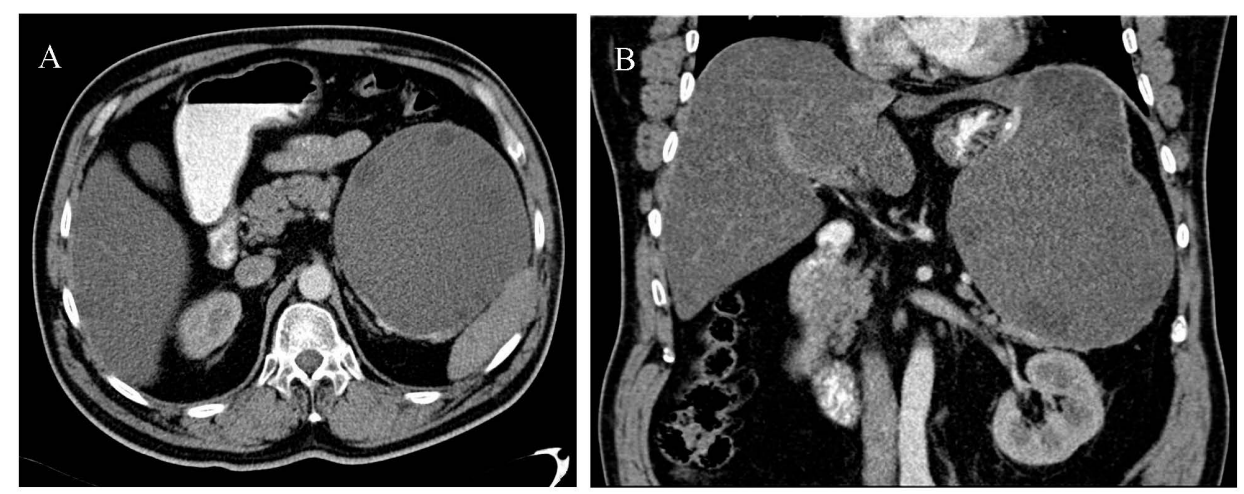

Figure 1. Computed tomography: (A) axial CT of bilobed hydatid cyst; (B) coronal CT of bilobed hydatid cyst.

Following the diagnosis, the patient underwent treatment with Albendazole, as recommended by the infectious disease physician.

\section{Surgical Management}

Due to the impressive size, form and content of the cyst, the patient was scheduled for classic surgery. The surgery was started with a median laparotomy. The organs of the peritoneal cavity and the mass described by computed tomography were explored. The exploration of the peritoneal cavity revealed intestinal loops mobilized in the lower abdomen by the anterior displacement of the posterior abdominal wall, with displaced and compressed spleen.

A completely retroperitoneal position of the hydatid cyst with a calcified upper pole was found, which seemed to start from the left hepatic lobe according to the CT description, corresponding to segment II of the liver, following a downward retroperitoneal direction, behind the lesser peritoneal sac (Figure 2). Next, the cyst had a downward direction, compressing the tail of the pancreas and also the spleen towards the left kidney, which it moved inferiorly. The lower pole of the hydatid cyst was located in the lower half of the abdomen, to the left side of the aorta, close to the bifurcation of the aorta, the position being completely retroperitoneal. The formation was not pulsating (according to the abdominal CT with contrast medium, there was no problem of a descending aortic aneurysm).

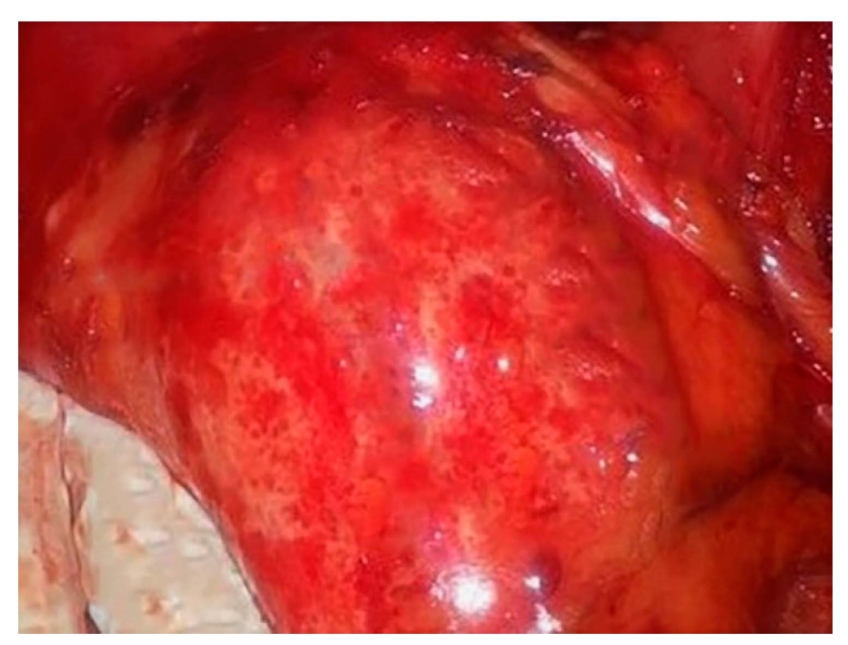

Figure 2. Intraoperative appearance of the cyst. 
The gastro-colic ligament was sectioned, and the great curvature of the stomach was de-vascularized in order to approach the middle region of the hydatid cyst. Following this, we isolated the middle area as well as the most inferior area of the tumor with multiple saline medical compresses. Inactivation of the cyst with saline liquid was performed by injecting it into the middle region of the formation, corresponding to the lesser peritoneal sac.

Saline liquid was also injected into the lower region of the cyst, because of its impressive size, to ensure that the hypertonic serum was distributed relatively evenly throughout the cavity. The lower pole of the cyst was easily palpable laterally and lower than the mesenteric insertion. We opened the cystic cavity in this lower left area. The content consisted of thousands of shriveled daughter cysts and viscous fluid, but without bilious content. The same was performed for the middle area of the cyst, corresponding to the lesser peritoneal sac.

Because the cyst was bilobed, the opening of the cyst in the middle and lower area allowed washing with I5\% saline liquid of the entire retroperitoneal cystic cavity from the upper to the lower part, allowing the mobilization of the cyst contents to the lower pole, where it could be evacuated (Figure 3). Lagrot type pericystectomy was attempted, but this was performed only on a limited area of the cyst due to bleeding and the risk of very high damage risk to important vascular structures-upper mesenteric artery, kidney artery and other collateral branches of the aorta. The area corresponding to the left hepatic lobe was hardened and calcified. Finally, the peritoneal cavity was rinsed.

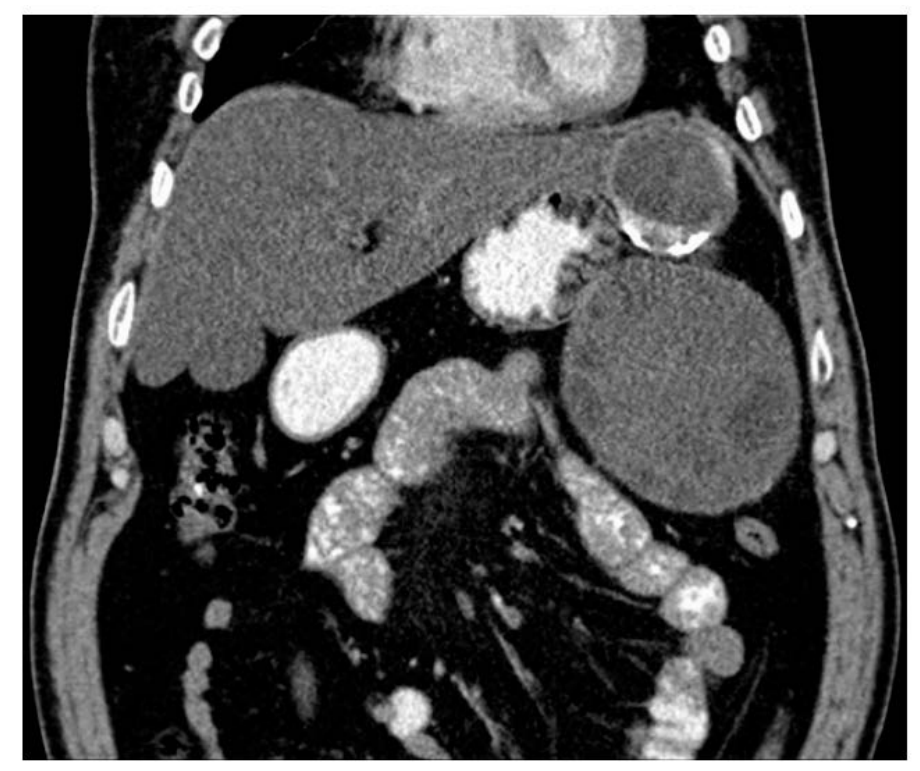

Figure 3. Computed tomography—-the appearance of the bilobed hydatid cyst (in the same CT).

Two large bore tubes exteriorized by epigastric laparostomy and two tubes exteriorized under the umbilical cord were installed inside the cyst on the midline incision. The subhepatic space and the bottom of the Douglas sac were also drained.

\section{Follow-Up}

The patient was discharged after 4 weeks with the drain tubes and came for regular check-ups. Intracavitary drainage tubes were maintained for a long time, but during regular check-ups, the tubes moved slightly, as they tend to adhere to intra-abdominal tissues. The epigastric drainages were first extracted after 2 months. 
During this period, a small amount of transparent liquid was found in the tube bag. The tubes at the lower pole of the hydatid cyst spontaneously protruded after about to weeks by cutting the skin by the thread with which they were sewn. At the patient's check-up presentation, the skin opening where the tubes were exteriorized was closed.

At five months after the surgery, the patient presented significant fatigue, subfebrile temperature $\left(37.8^{\circ} \mathrm{C}\right)$ and sweating, with the general condition slowly degrading during the previous two weeks. Subsequently, the patient was re-admitted to our department and underwent abdominal computed tomography, which revealed a hydro-aeric image with calcified walls of oval shape with diameters of $8.2 \times 4.6 \mathrm{~cm}$-interpreted as a remaining cavity (Figure 4 ).

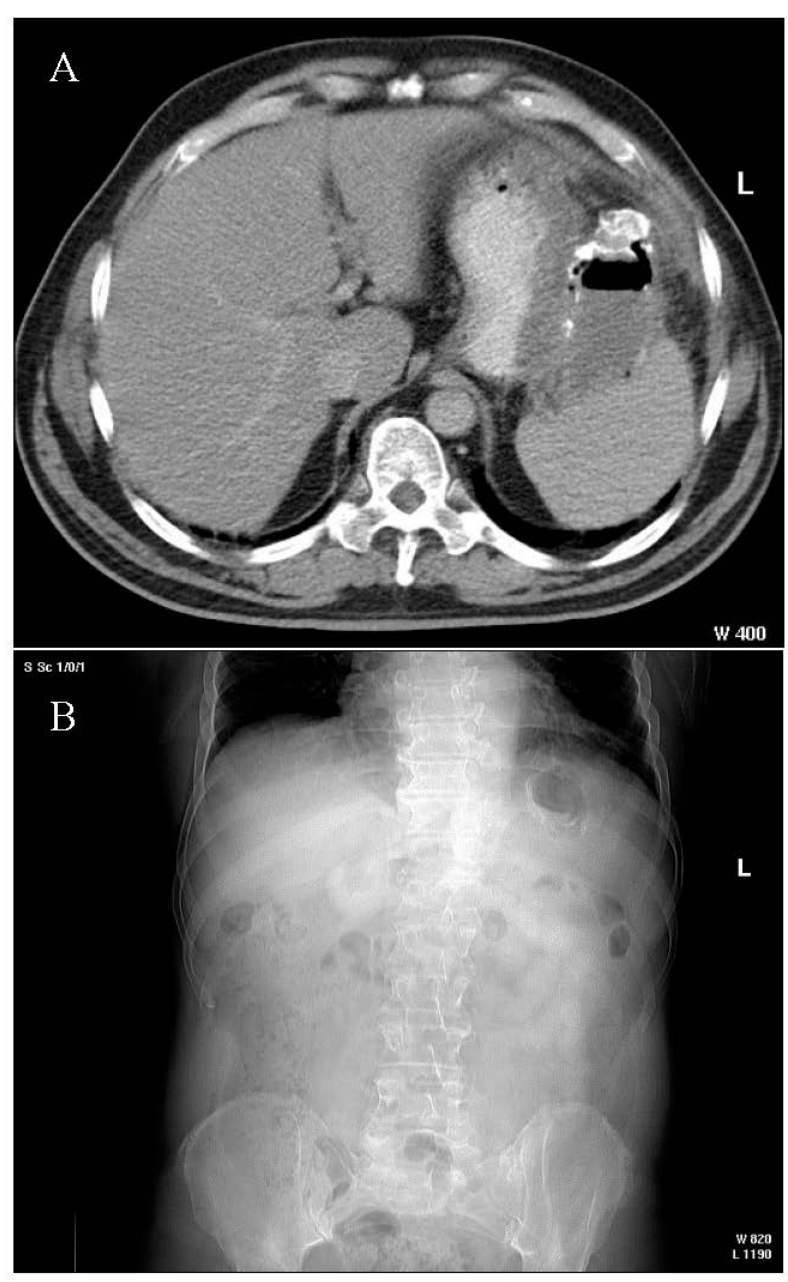

Figure 4. (A) The coronal CT image of calcified remaining cavity; (B) the axial CT image of calcified remaining cavity.

We decided to perform a surgical reintervention to drain the remaining cavity. A median incision was made, followed by adhesiolysis; the remaining cystic cavity was reduced in volume, having the appearance of an inflammatory block with thick walls, impossible to dissect. We decided to insert a tube inside the cavity checking the lower position of the drainage tube radiologically. The remaining cavity was rinsed with betadine and serum through the drainage. Afterwards, the abdominal wall was sutured. A bacteriological exam of the aspirated fluid from the cavity showed that no germs grew on the culture medium. 
The patient's postoperative evolution was favorable, with the remission of symptoms; the drain tube was removed after 4 weeks. The patient resumed his work activity after 6 weeks and did not return for follow-up visits.

During an evaluation performed after 6 years for the left inguinal hernia, the abdominal and pelvis CT with contrast medium was repeated and showed the following: liver with significant hepatic steatosis, pancreas within normal limits, adrenal glands within normal limits, left and right kidney with normal function and no fluid collections at abdominal or pelvic level.

The evolution of our case was finally favorable, but it confirmed that the evolution of large hydatid cysts can be associated with a wide range of postoperative complications, as in our situation, with difficult closure of the remaining cavity, also possibly due to the bilobed appearance of the cyst.

\section{Discussion}

Hydatid disease is characterized by an increased frequency worldwide in so-called "endemic" areas and is particularly severe due to the long absence of clinical signs and its detection in late stages (similar to neoplastic diseases).

Hydatid cysts in the retroperitoneal space are frequently asymptomatic until they grow and produce symptomatology caused by high intracystic pressure, secondary infection, break of the walls and allergy. When they require surgery, this may be difficult to perform, with high operative risks and the need for re-interventions.

There are great controversies regarding the management of liver hydatidosis, as various therapy options are available.

\section{Surgery}

Classic Surgery

For more than a hundred years, the recommended method for hepatic hydatid cysts has been surgical excision through a conservative or radical operation, and surgery remains the cornerstone of hydatid liver cyst care. Surgery can remove the cyst and pericardium along with its contents, which leads to complete healing. Classic surgical treatment includes radical methods (cystectomy with total precystectomy and partial liver resection) or conservative techniques (cystectomy with partial Lagrot precystectomy and drainage of the cystic cavity). The more radical the method, the greater the operative risk, but also the likelihood of recurrence is lower $[8,9]$.

Open surgery is indicated for big hepatic cysts, superficially located hepatic cysts that will naturally rupture as a consequence of contusions; cysts that communicate with bile ducts; cysts that compress adjacent structures; cysts with contaminated content; and those in the lungs, kidneys, central nervous system, bones and any organ $[\mathrm{IO}, \mathrm{II}]$. It is the method of choice in case of cysto-biliary fistula and old, voluminous cysts.

Although pericystectomy leads to complete healing with a low risk of recurrence, Schmidt-Matthiesen, O. Schott and A. Encke in a comparative retrospective paper concluded that because pericystectomy is followed by higher morbidity, it is preferable to use conservative procedures [12]. We have to suture visible liver communication between cyst and biliary ducts, internal or external drainage (evacuation of daughter cysts through choledochotomy and choledochal Kehr drainage) of the communication [13]. 
According to C. Dziri and A. Fingerhut, there are many postoperative complications, such as residual cavity, bleeding, liver bile leaks consecutive to pericystectomy, bile or blood collections and communications between cyst and biliary ducts; the percentage found in their paper was between I2 and 26\% [14].

\section{Laparoscopic Surgery}

Laparoscopic surgery is suitable for uncomplicated hydatid cysts, localized superficially in the liver segments easily accessible by this method (especially liver segments III, IVb, V and possibly VI-according to Couinaud's classification), but hydatid cysts can also be accessed on other segments of the liver [I5].

However, in the case of the localization of the hepatic hydatid cyst in segments VII-VIII, classical surgery is preferable. Laparoscopic surgery is also not indicated in cystic-biliary communications [3]. It is useful to perform endoscopic retrograde cholangiopancreatography before the operation to diagnose a possible cysto-biliary fistula and eliminate the discomfort of a conversion to classic surgery [16].

\section{Minimally-Invasive Therapy}

Ultrasound-guided percutaneous Puncture, Aspiration, Injection and Re-aspiration (PAIR) is recommended by the World Health Organization (WHO), being feasible in types CEI (simple cyst, unilocular), $\mathrm{CE}_{2}$ (multivesicular, multiseptated) and $\mathrm{CE}_{3}$ (floating membranes) from the WHO classification of hydatid cysts $[6,8]$.

The statistics presented by Yagci on a group of 355 patients showed complications in $28.1 \%$ of cases in open surgery, ${ }^{3} 3.3 \%$ of cases in laparoscopic surgery and $9.2 \%$ of cases in percutaneous treatment [13]. According to Duță et al., in the case of a cyst that is difficult to operate because of its position, an ultrasound guided puncture is very useful [i7]. The PAIR technique is generally suitable for smaller cysts, although the classification described by the WHO includes all dimensions. It is also not indicated in cystic-biliary communications.

In his retrospective paper, Gupta compared three groups of patients: the first group included 52 cases with PAIR, the second group 6I cases with radical approaches and the third group 33 with conservative approaches $[2, \mathrm{I} 7]$. He concluded that it is important to select only patients with Gharbi's I and II type of cysts, (CEI and $\mathrm{CE}_{2}$ from WHO classification) for a safe PAIR approach [17,I8].

\section{Conclusions}

Our case presentation confirmed that the classic surgical approach was the appropriate choice, considering the impressive size of the hydatid cyst and its content. The prevention of cyst rupture is the secret to surgical success; for allergic syndrome prevention, dexamethasone could be used before cyst resection [19]. Bilobed cysts and those with calcified walls may cause problems during the closure of the remaining cavity.

For complicated cysts, the preferred treatment is radical or conservative surgery, but for uncomplicated cysts, minimally invasive treatment is an appropriate choice. Albendazole can be used before and after the surgical approach to prevent the risk of recurrence.

Author Contributions: Conceptualization, M.M., S.P. and S.O.; Methodology, S.O.; Investigation, M.M. and S.P.; Writing-Original Draft Preparation, M.M.; Writing-Review and Editing, M.M., S.P. and S.O.; Supervision, S.O. 
Funding: This research received no external funding.

Conflicts of Interest: The authors declare no conflict of interest.

\section{References}

I. Devi, M.A.; Venumadhav, T.; Sukanya, B.; Manmada, R.T.; Gopal, P.; Rammurti, S. Role of Imaging in Diagnosis, Predicting Biological Activity and in Treatment Plan of Hydatid Disease. Open J. Intern. Med. 2018, 8, 177-195. [CrossRef]

2. Gupta, N.; Javed, A.; Puri, S.; Jain, S.; Singh, S.; Agarwal, A.K. Hepatic Hydatid: PAIR, Drain or Resect? J. Gastrointest. Surg. 2011, 15, I829-1836. [CrossRef] [PubMed]

3. Zaharie, F.; Bartos, D.; Mocan, L.; Zaharie, R.; Iancu, C.; Tomus, C. Open or laparoscopic treatment for hydatid disease of the liver? A ro-year single-institution experience. Surg. Endosc. 2013, 27, 2110-2116. [CrossRef] [PubMed]

4. Azlaf, R.; Dakkak, A. Epidemiological study of the cystic echinococcosis in Morocco. Vet. Parasitol. 2006, 137, 83-93. [CrossRef] [PubMed]

5. Çabadak, H.; Erbay, A.; Aypak, A.; Tekçe, A.Y.T.; Şen, S. Giant hydatid cyst of spleen: A case report. Trop. Doct. 2009, 39, 248-249. [CrossRef] [PubMed]

6. World Health Organization, Department of Communicable Disease. Puncture, Aspiration, Injection, Re-aspiration: An Option for the Treatment of Cystic Echinococcosis. WHO/CDS/CSR/APH/2001.6 Bull. World Health Organ. 2001, 6, I-44.

7. WHO Informal Working Group on Echinococcosis. Guidelines for treatment of cystic and alveolar echinococcosis in humans. Bull. World Health Organ. 1996, 74, 23I-242.

8. Moro, P.L.; Cantey, P. Echinococcus Species. In Principles and Practice of Pediatric Infectious Diseases, 5 th ed.; Elsevier: Philadelphia, PA, USA, 2018; pp. I404-I4IO. [CrossRef]

9. Eckert, J.; Deplazes, P. Alveolar echinococcosis in humans: The current situation in central Europe and the need for countermeasures. Parasitol. Today 1999, 15, 315-319. [CrossRef]

Io. Frider, B.; Larrieu, E. Treatment of liver hydatidosis: How to treat an asymptomatic carrier? World J. Gastroenterol. 2010, 16, 4I23-4I29. [CrossRef] [PubMed]

II. Buttenschoen, K.; Buttenschoen, D.C. Echinococcus granulosus infection: The challenge of surgical treatment. Langenbeck's Arch. Surg. 2003, 388, 218-230. [CrossRef] [PubMed]

I2. Schmidt-Matthiesen, A.; Schott, O.; Encke, A. Surgery and long-term follow-up of hepatic echinococcosis outside endemic regions. Z. Gastroenterol. 2002, 40, 5I-57. [CrossRef] [PubMed]

13. Yagci, G.; Ustunsoz, B.; Kaymakcioglu, N.; Bozlar, U.; Gorgulu, S.; Simsek, A.; Akdeniz, A.; Cetiner, S.; Tufan, T . Results of surgical, laparoscopic, and percutaneous treatment for hydatid disease of the liver: Io Years experience with 355 patients. World J. Surg. 2005, 29, 1670-1679. [CrossRef] [PubMed]

I4. Dziri, C.; Haouet, K.; Fingerhut, A. Treatment of hydatid cyst of the liver: Where is the evidence? World J. Surg. 2004, 28, 73I-736. [CrossRef] [PubMed]

I5. Khoury, G.; Abiad, F.; Geagea, T.; Nabout, G.; Jabbour, S. Laparoscopic treatment of hydatid cysts of the liver and spleen. Surg. Endosc. 2000, 14, 243-245. [CrossRef] [PubMed]

16. Hamza, A.; Krasniqi, A.; Sada, F.; Zejnullahu, V.; Bicaj, B. ERCP treatment of obstructive jaundice caused by hydatid cyst in extrahepatic ducts 13 years after liver hydatid endocystectomy. A case report. Int. J. Surg. Case Rep. 2020, 74, 38-4I. [CrossRef] [PubMed]

17. Duta, C.; Pantea, S.; Lazar, C.; Salim, A.; Barjica, D. Minimally invasive treatment of liver hydatidosis. J. Soc. Laparoendosc. Robot. Surg. 2016, 20, e2016.00oo2. [CrossRef] [PubMed]

I8. Gharbi, H.A.; Hassine, W.; Brauner, M.W.; Dupuch, K. Ultrasound examination of the hydatic liver. Radiology 1981, 139, 459-463. [CrossRef] [PubMed]

19. Zhao, Q.; Luo, J.; Zhang, Q.; Leng, T.; Yang, L. Laparoscopic surgery for primary ovarian and retroperitoneal hydatid disease. Medicine 2018, 97, e9667. [CrossRef] [PubMed]

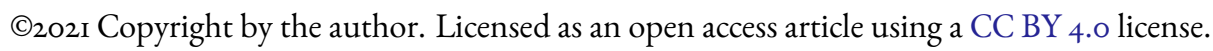

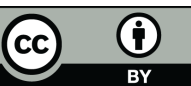

